\title{
Some effects of encoding, codability, and exposure upon recognition memory
}

\author{
PAT-ANTHONY FEDERICO \\ Navy Personnel Research and Development Center, San Diego, California 92152
}

\begin{abstract}
The primary purpose of this research was to investigate whether verbal and imaginal encoding interact with stimulus codability and exposure either to enhance or to retard recognition memory for shapes. The secondary purpose was to test the conceptual coding hypothesis (Ellis, 1972). Two multivariate factorial experiments were conducted. In the first study, encoding was a between-groups factor; in the second study, encoding was a within-subjects factor. The other within-subjects factors investigated in both experiments were codability, encoding exposure, and recognition exposure. It was found that the interaction of encoding and codability was significant. The conceptual coding hypothesis was somewhat substantiated by these results.
\end{abstract}

There is little doubt that human subjects can recognize objects or shapes by encoding them verbally or imaginally. However, a question that remains is this: How do these two encoding strategies interact with various stimulus characteristics either to enhance or to retard recognition performance? The limited data pertinent to this question appear to be contradictory. Several studies exploring the effects of distinctive verbal encoding on recognition memory for shapes have yielded inconsistent results. The effects of verbal encoding on recognition memory for shapes have been both negative (Bahrick \& Boucher, 1968; Hake \& Eriksen, 1956; Prentice, 1954; Santa \& Ranken, 1968; Vanderplas \& Garvin, 1959b) and positive (Clark, 1965; Daniel \& Ellis, 1972; Ellis \& Daniel, 1971; Ellis \& Homan, 1968; Ellis \& Muller, 1964; Federico \& Montague, 1975; Kelly \& Martin, 1974; Price \& Slive, 1970; Ranken, 1963; Santa \& Ranken, 1972).

Some of these findings led to the conceptual coding hypothesis (Ellis, 1972; Ellis \& Muller, 1964; Ellis \& Shumate, 1973). It assumes that either encoding strategy, verbal or imaginal, produces its greatest relative success with stimuli that are fractionated, impoverished, or difficult to code. The consequence of associating verbal responses to shape stimuli is dependent upon the initial codability of the stimuli, with the relative effects of verbal encoding being greater with less codable stimuli. Codability has been defined as "a generic term referring to the class of stimulus properties-e.g., physical, associative, linguistic-which control the ease with which stimulus items may be placed in memory" (Ellis \& Shumate, 1973, p. 71). It is' alleged that the more difficult a shape is to encode initially, the greater is the relative contribution of a verbal code in enhancing

Opinions or assertions contained herein are those of the author and are not to be construed as official or reflecting the views of the Department of the Navy. Requests for reprints should be sent to Pat-Anthony Federico, Navy Personnel Research and Development Center (NPRDC Code P304), San Diego, California 92152. recognition. Emphasis is placed on the relative contribution, since stimuli that are at first easy to encode will probably be recognized regardless of the presence of a verbal code. More generally, this hypothesis implies that as memory is strained because of complex stimuli or brief stimulus exposures, the availability of a verbal code becomes increasingly important in determining recognition performance. The present article reports two experiments that shed additional light on how encoding affects recognition memory for shapes. The primary purpose was to investigate whether verbal and imaginal encoding interact with salient stimulus characteristics (i.e., codability and exposure) either to enhance or to retard recognition performance. The secondary purpose was to test the conceptual coding hypothesis.

\section{EXPERIMENT 1}

\section{Method}

Subjects. Subjects were from two companies of Navy recruit trainees who were stationed at the Naval Training Center, San Diego. These recruits had been randomly assigned to their training companies, which consisted of 54 and 59 members, respectively. Subjects who did not follow instructions or did not finish the task were discarded. This process, together with randomly discarding other subjects, produced two groups of 47 subjects each.

Design. A 2 by 2 by 2 by 2 multivariate factorial design was used with 10 criterion responses as dependent variates. There was one between-groups factor, verbal (VB) or imaginal (IM) encoding strategy, and three within-groups factors: (1) stimulus codability, high or low; (2) encoding exposure, short or long; and (3) recognition exposure, short or long. The 10 criterion responses consisted of subjects' confidence ratings in their judgments of recognition of 10 high-codability $(\mathrm{HC})$ or 10 lowcodability (LC) random shapes.

Independent variables manipulation. Encoding strategy was manipulated by instructional set. The verbal instructions asked the subjects to describe, name, or label some familiar object or situation of which the projected random shapes reminded them. The imaginal instructions asked the subjects to picture, imagine, or visualize the random shapes in their mind's eye. A procedure was followed that forced subjects to comply with the instructional sets: They were required to write a clear verbal encoding 
of each shape or to draw a detailed imaginal encoding of each shape. Obviously, this cannot completely prevent parallel verbal and imaginal encoding, but it can produce a difference in encoding emphasis.

Codability was manipulated as follows: Stimuli were random shapes from a pool scaled according to association value (A) (Vanderplas \& Garvin, 1959a), which varied in complexity (the number of points that determine inflections on the perimeter of the shapes). There is an inverse relationship between shape complexity and the number, content, and heterogeneity of associations. Consequently, $40 \mathrm{HC}$ stimuli were of low complexity (4- and 6-point shapes) and high association value (A $>42$ and $A>38$, respectively, and 40 LC stimuli were of high complexity (24- and 16-point shapes) and low association value ( $A<40$ and $A<38$, respectively). In the Vanderplas and Garvin (1959a) identification scheme, the 4- and 6-point shapes were specified as numbers 1-20 and the 24- and 16-point shapes were specified as the numbers 11-30. It was presumed on the basis of Vanderplas and Garvin's data that the sets of 4- and 24-point shapes were equivalent in codability to the sets of 6- and 16-point shapes, respectively.

Short encoding and recognition exposure was defined as $.5 \mathrm{sec}$, and long encoding and recognition exposure was defined as $5.0 \mathrm{sec}$. The stimuli consisted of $35-\mathrm{mm}$ slides of black random shapes on transparent backgrounds. These stimuli were displayed by a Kodak Ektagraphic slide projector (Model AF-2), using an f3.5 4- to 6-in. zoom Ektanar lens and a 400-W Sylvania bulb. Exposure and interstimulus interval were controlled by Hunter decade interval timers (Models 111-B and $-\mathrm{C}$, respectively). These were interconnected with the projector and a Mackenzie Adjusto-Dissolve (Model AD-2). A Wallmaster Radient daylight screen was positioned in front of a room of sufficient size to accommodate one of the two groups at a time. The projector-to-screen distance was $24 \mathrm{ft} 6 \mathrm{in}$. The projected stimulus shapes were approximately $6 \mathrm{ft}$ wide and $4 \mathrm{ft}$ high. Ambient illumination within the room was $3.0 \mathrm{fc}$.

Dependent variates measurement. Recognition memory for random shapes was defined as follows: Subjects were shown $\mathrm{HC}$ and LC random shapes. After having seen each subset, they were required to select those shapes they had seen before from among similar shapes in two larger sets that included some they had not seen before. As each stimulus was projected, subjects were asked to rate their confidence in their recognition judgment. They checked either "seen before" or "not seen before" and $20 \%, 40 \%, 60 \%, 80 \%$, or $100 \%$; these were scored plus or minus and $1,2,3,4$, or 5 , respectively.

Procedure. Each cluster of $204-, 6-, 16-$, and 24-point shapes was divided into 10 even- and 10 odd-numbered stimuli. From each of these four even and four odd groupings of 10 shapes, 5 shapes were randomly selected to be target stimuli. The remaining five shapes within each even or odd grouping served as distractor stimuli during recognition testing.

Target shapes were displayed for either short or long exposures. Within each encoding exposure, subjects were presented $\mathrm{HC}$ and LC shapes. During later recognition testing, these stimuli were displayed for short or long exposures. The presentation sequence of short or long encoding exposure was counterbalanced across the two different encoding groups. Similarly, the presentation sequence of HC or LC shapes was counterbalanced across encoding exposures. Also, the presentation sequence of short or long recognition exposure was counterbalanced across HC and LC stimuli. All of this was feasible and facilitated by counterbalancing the presentation sequence of even- or odd-numbered HC and LC stimuli across encoding exposures.

When all subjects understood the instructions, either five $\mathrm{HC}$ or five LC shapes were shown in random order for a short or a long encoding exposure, using an interstimulus interval of $20 \mathrm{sec}$. Within this interval, subjects were to write or to draw their encoding for each stimulus. The subjects were then instructed to turn to the next numbered blank page in the response booklet to ensure that the encodings were synchronized with the proper target stimulus. Following the encoding of these five HC or LC shapes, subjects were given an interpolated task that consisted of canceling from the page of random numbers as many even or odd one-, two-, three-, or five-digit random numbers as they could in $1 \mathrm{~min}$. Subjects were then shown in random order 10 even- or odd-numbered HC or LC shapes, using either a short or a long recognition exposure. These random sequences of 10 stimuli included target as well as distractor shapes. During recognition testing, the interstimulus interval was $10 \mathrm{sec}$. Subsequently, the subjects were given $5 \mathrm{~min}$ to complete an open-ended postexperimental questionnaire.

\section{Results}

Under the assumptions of multivariate normality and homogeneity of group dispersions, a 2 by 2 by 2 by 2 multivariate analysis of variance with 10 dependent variates and 47 replicates was computed on the subjects' scored confidence ratings. The interaction of encoding and codability was significant at the .05 level $[\mathrm{U}(10,1,736)=.97, \mathrm{~F}(10,727)=1.88, \lambda=.29$, $\mathrm{F}(60,1054)=8.94, \tau=1.69]$. Three discriminant functions accounted for $100 \%$ of the trace. The centroids for these treatment combinations, computed relative to the first discriminant function, were $10.20,6.43,9.52$, and 1.49 for VB-HC, VB-LC, IM-HC, and IM-LC, respectively. The discriminant-function weights and productmoment correlations indicated the consequential contributors among the encoded and distractor shapes to the separation along the first discriminant function of the treatment combinations produced by the interaction of encoding and codability. Almost twice as many encoded shapes as distractor shapes contributed significantly to the separation among treatment combinations along the first discriminant function. Considering the initial $69.31 \%$ of the variance associated with the first discriminant function $\left[\chi^{2}(22)=282.59\right]$, the major portion of the interactive effect of encoding and codability was due to differences in confidence ratings attributed to the encoded shapes. Subsequently, the Scheffé (1959) procedure was followed for each dependent variate. Verbal and imaginal encoding for $\mathrm{HC}$ shapes produced essentially equivalent recognition performance. This, in turn, was better than verbal encoding for LC shapes. However, all of these conditions resulted in recognition performance significantly $(p<.01)$ superior to that of imaginal encoding for LC shapes. The means for each encoded and distractor stimulus showed that this interactive effect of encoding and codability was due primarily to the differences in the subjects' confidence ratings attributed to their judgments of the encoded shapes. This substantiated what was indicated by the weights and correlations for the first discriminant function.

A content analysis was conducted of the codes generated by the subjects in the verbal encoding group to facilitate their recognition memory. Considerably more 
of them used three or more words to mediate their responses to LC stimuli than to $\mathrm{HC}$ stimuli. These subjects seemed to take into consideration the details, irregularity, and bizarreness of LC shapes. In addition, there was more homogeneity among the subjects' encodings and less differentiation or qualification by the use of adjectives or adverbs for HC shapes than for LC shapes. An analysis of the codes produced by the subjects in the imaginal encoding group indicated that $\mathrm{HC}$ stimuli were reproduced with more accuracy than were LC stimuli. Apparently, the high complexity of the LC shapes relative to the $\mathrm{HC}$ shapes interfered with the quality of the subjects' imaginal encodings.

\section{EXPERIMENT 2}

\section{Method}

Subjects. Subjects were from an additional two companies of Navy recruit trainees who were also stationed at the Naval Training Center, San Diego. Applying the same criteria used in Experiment 1 produced two groups of 53 subjects each.

Design. The design was a 2 by 2 by 2 by 2 by 2 multivariate factorial design with four dependent variates. Four of these factors were identical to those in Experiment 1 and were withinsubjects manipulations: (1) encoding strategy, (2) stimulus codability, (3) encoding exposure, and (4) recognition exposure. The fifth factor was order of instructional set (i.e., verbal-imaginal or imaginal-verbal), which was a between-groups manipulation.

Independent variables manipulation. Encoding strategy, encoding exposure, and recognition exposure were defined as in Experiment 1 . Stimulus codability was manipulated in a manner similar to the first study; however, $32 \mathrm{HC}$ stimuli were of low complexity (4- and 6-point shapes) and high association value (A $>44$ and A $>38$, respectively), and 32 LC stimuli were of high complexity (24- and 16-point shapes) and low association value ( $\mathrm{A}<38$ and $\mathrm{A}<36$, respectively). In the Vanderplas and Garvin (1959a) identification scheme, the 4- and 6-point shapes were specified as numbers 1-16, and the 24- and 16-point shapes were specified as numbers 15-30. Again, it was presumed that the sets of 4- and 24-point shapes were equivalent in codability to the sets of 6-and 16-point shapes, respectively.

Dependent variates measurement. Recognition memory for the random shapes was defined as in Experiment 1. Also, subjects' responses were scored in a similar manner.

Procedure. Each cluster of 16 4-, 6-, 16-, and 24-point shapes was divided into 8 even- and 8 odd-numbered stimuli. Each of these four even and odd groupings of eight shapes was then divided into two subgroupings of four shapes. From each of these subgroups, two were randomly selected to be target stimuli. The remaining two shapes within each subgroup served as distractor stimuli during subsequent recognition testing.

The rest of the procedure was similar to Experiment 1.

\section{Results}

A 2 by 2 by 2 by 2 by 2 multivariate analysis of variance with four dependent variates and 53 replicates was computed on the subjects' scored confidence ratings. The interaction of encoding and codability was significant at the .05 level $[\mathrm{U}(4,1,1664)=.99, \mathrm{~F}(4,1661)$ $=2.57, \lambda=.72, F(24,2428)=12.11, \tau=.37]$. Three discriminant functions accounted for $100 \%$ of the trace for these treatment combinations. The centroids for these treatment combinations computed relative to the first discriminant function were $6.77,6.07,7.08$, and 2.92 for VB-HC, VB-LC, IM-HC, and IM-LC, respectively. The first discriminant function alone accounted for $86.35 \%$ of the variance $\left[\chi^{2}(10)=234.05\right]$. The discriminant-function weights and product-moment correlations indicated that the encoded and distractor shapes contributed about equally well to the significant separation among treatment combinations along the first discriminant function. The Scheffe procedure was applied to each dependent variate. As in Experiment 1, verbal encoding for $\mathrm{HC}$ and $\mathrm{LC}$ shapes and imaginal encoding for $\mathrm{HC}$ shapes generally produced equivalent recognition performance. Yet, these treatment conditions resulted in significantly $(p<.01)$ better recognition performance than did imaginal encoding for LC shapes.

Content analyses were conducted of the codes generated by subjects who received both instructional sets. The results were similar to the findings of the content analyses performed for Experiment 1.

\section{DISCUSSION}

In Experiments 1 and 2, recognition with verbal encoding for HC and LC shapes and imaginal encoding for HC shapes was superior to that with imaginal encoding for LC shapes. This demonstrates the differential effect of verbal encoding, in that recognition memory for LC shapes improved more, or was facilitated more, than recognition for $\mathrm{HC}$ shapes. This result substantiated the conceptual coding hypothesis (Ellis, 1972; Ellis \& Muller, 1964; Ellis \& Shumate, 1973) in the sense that the consequence of associating verbal responses to shape stimuli was dependent upon the initial codability of the stimuli, with the relative effects of verbal encoding being greater with the less codable stimuli.

The perceptual organization hypothesis is similar to the conceptual coding hypothesis; however, the former goes beyond the latter by suggesting "an elaborative construction of the visual representation" (Santa, 1975, p. 287). This concept of perceptual organization implies that a verbal code produces a perceptual restructuring of the shape in terms of the association(s) elicited by the verbal code itself. This cognitive restructuring could have induced the global perception of an LC shape as an integrated whole, which could have later facilitated its redintegration and recognition. That is, a verbal code could have not only influenced the encoding of a stimulus (Nicosia \& Santa, 1975), but also solidified a cognitive representation of a stimulus by linking its diverse characteristics to a single reference-the verbal code. This could have stabilized or integrated the stored representation of an LC shape to such an extent that its salient features were closely linked. The occurrence of one distinctive feature in memory could have easily evoked by means of the verbal code the rest of the representation of this specific LC shape. Thus, this could have accounted for the beneficial effect of verbal encoding, as opposed to imaginal encoding, upon recognition memory for LC shapes.

The above finding for Experiments 1 and 2 corroborated the results of those studies that established positive effects of verbal encoding upon recognition memory for shapes (Clark, 1965; Daniel \& Ellis, 1972; Ellis \& Daniel, 1971; Ellis \& Homan, 1968; Ellis \& Muller, 1964; Federico \& Montague, 1975; Kelly \& Martin, 1974; Price \& Slive, 1970; Ranken, 1963; Santa \& Ranken, 1972). It seemed that this verbal encoding effect could be attributed primarily to processes occurring during the acquisition phase of the recognition task. Verbal encoding not only 
could have provided stable, readily made representations of LC shapes, but also could have encouraged a visual search for critical or distinctive features. This interpretation implied that verbal encoding elicited by or associated with LC shapes helped to distinguish among them and therefore enhanced their recognition by minimizing confusion among them. The discovery that LC shapes were better recognized under verbal encoding than under imaginal encoding, nevertheless, did not corroborate the results of those studies that established negative effects of verbal encoding upon recognition memory for shapes (Bahrick \& Boucher, 1968; Hake \& Eriksen, 1956; Prentice, 1954; Santa \& Ranken, 1968; Vanderplas \& Garvin, 1959b).

Since it was established in Experiments 1 and 2 that verbal and imaginal encoding of HC shapes produced equivalent recognition performance, it could be inferred that $\mathrm{HC}$ shapes can be encoded verbally or imaginally without affecting their recognition. This suggested, too, that HC shapes, unlike LC shapes, can be stored as simple images (Clark, 1965). Some evidence that seemed to imply this was that the imaginal codes were drawn by the subjects with more accuracy for HC shapes than for LC shapes. This finding supported the assertions that (1) verbal encoding need not be resorted to if the stimuli are simple shapes that can be stored as uncoded images, and (2) imaginal encoding can be the basis of the distinctiveness and recognizability of simple shapes (Paivio, 1971). Possibly, because of their greater concreteness relative to the more abstract LC shapes, HC shapes could have readily allowed images of their contours to be recognized more effectively than contours of LC shapes. Consequently, imaginal encoding was just as effective as verbal encoding for $\mathrm{HC}$ shapes. This result and its corresponding interpretation, however, does not completely agree with the explanation that the primary memory mechanism of a shape is imaginal rather than verbal (Posner \& Konick, 1966).

\section{REFERENCES}

BAhrick, H. P., \& Boucher, B. Retention of visual and verbal codes of the same stimuli. Journal of Experimental Psychology, $1968,78,417-422$.

ClARK, H. J. Recognition memory for random shapes as a function of complexity, association value, and delay. Journal of Experimental Psychology, 1965, 69, 590-595.

Daniel, T.C., \& Elllis, H. C. Stimulus codability and longterm recognition memory for visual form. Journal of Experimental Psychology, 1972, 93, 83-89.

Ellis, H. C. Verbal processes in the encoding of visual pattern information: An approach to language, perception, and memory. In M. E. Meyer (Ed.), Symposium on cognitive learning. Bellingham: Western Washington State College Press, 1972.

Ellis, H. C., \& Daniel, T. C. Verbal processes in long-term stimulus recognition memory. Journal of Experimental Psychology, 1971, 90, 18-26.
Ellis, H. C., \& Homan, L. E. Implicit verbal responses and the transfer of stimulus predifferentiation. Journal of Experimental Psychology, 1968, 76, 486-489.

Ellis, H. C., \& Muller, D. G. Transfer in perceptual learning following stimulus predifferentiation. Journal of Experimental Psychology, 1964, 68, 388-395.

Ellis, H. C., \& Shumate, E. C. Encoding effects of response belongingness and stimulus meaningfulness on recognition memory of trigram stimuli. Journal of Experimental Psychology, 1973, 98, 70-78.

Federico, P-A., \& Montague, W. E. Recognition memory as a function of encoding strategy and stimulus codability. Journal of Experimental Psychology: Human Learning and Memory, $1975,1,680-688$.

Hake, H. W., \& Eriksen, C. W. Role of response variables in recognition and identification of complex visual forms. Journal of Experimental Psychology, 1956, 52, 235-243.

Kelly, R. T., \& Martin, D. W. Memory for random shapes: A dual-task analysis. Journal of Experimental Psychology, 1974, 103, 224-229.

Nicosia, G. J., \& SANTA, J. L. Words and the reproduction of nonsense things. Journal of Experimental Psychology: Human Learning and Memory, 1975, 104, 369-375.

Paivio, A. Imagery and verbal processes. New York: Holt, Rinehart, \& Winston, 1971.

Posner, M. I., \& Konick, A. F. Short-term retention of visual kinesthetic information. Organizational Behavior and Human Performance, 1966, 1, 71-86.

Prentice, W. C. H. Visual recognition of verbally labeled figures. American Journal of Psychology, 1954, 67, 315-320.

Price, R. H., \& Slive, A. B. Verbal Processes in shape recognition. Journal of Experimental Psychology, 1970, 83, 373-379.

RANKEN, H. B. Effects of name learning on serial learning, position learning, and recognition learning with random shapes. Psychological Reports, 1963, 13, 663-678.

Santa, J. L. Verbal coding and redintegrative memory for shapes. Journal of Experimental Psychology: Human Learning and Memory, 1975, 104, 286-294.

SANTA, J. L., \& RANKEN, H. B. Language and memory: Redintegrative memory for shapes facilitated by naming. Psychonomic Science, 1968, 13, 109-110.

Santa, J. L., \& Ranken, H. B. Effects of verbal coding on recognition memory. Journal of Experimental Psychology, 1972, 93, 268-278.

SchefFE, H. The analysis of variance. New York: Wiley, 1959.

VANDE RPlas, J. M., \& Garvin, E. A. The association value of random shapes. Journal of Experimental Psychology, 1959, 57, 147-154. (a)

Vanderplas, J. M., \& Garvin, E. A. Complexity, association value, and practice as factors in shape recognition. Journal of Experimental Psychology, 1959, 57, 155-163. (b)

(Received for publication June 5, 1980.) 